\title{
Structure and binding properties of Pangolin-CoV Spike glycoprotein inform the evolution of SARS- CoV-2.
}

\section{Antoni G. Wrobel ( $\square$ antoni.wrobel@crick.ac.uk)}

The Francis Crick Institute https://orcid.org/0000-0002-6680-5587

Donald J. Benton ( $\boldsymbol{D}$ donald.benton@crick.ac.uk)

The Francis Crick Institute https://orcid.org/0000-0001-6748-9339

Pengqi Xu

Precision Medicine Center, The 10 Seventh Affiliated Hospital, Sun Yat-sen University, Shenzhen

Annabel Borg

The Francis Crick Institute

Chloë Roustan

The Francis Crick Institute

\section{Stephen R. Martin}

The Francis Crick Institute

\section{Peter B. Rosenthal}

The Francis Crick Insitute https://orcid.org/0000-0002-0387-2862

John J. Skehel

The Francis Crick Institute https://orcid.org/0000-0002-8519-9404

Steven J. Gamblin ( $\nabla$ steve.gamblin@crick.ac.uk )

The Francis Crick Insitute https://orcid.org/0000-0001-5331-639X

\section{Research Article}

Keywords: SARS-CoV-2, electron cryomicroscopy, pangolin

Posted Date: September 29th, 2020

DOI: https://doi.org/10.21203/rs.3.rs-83072/v1

License: (c) (i) This work is licensed under a Creative Commons Attribution 4.0 International License. Read Full License

Version of Record: A version of this preprint was published at Nature Communications on February 5th, 2021. See the published version at https://doi.org/10.1038/s41467-021-21006-9. 

1 Structure and binding properties of Pangolin-CoV Spike glycoprotein inform the evolution of SARS-CoV-2

Antoni G. Wrobel\#*1, Donald J. Benton\#*1, Pengqi Xu' ${ }^{4,1}$, Annabel Borg ${ }^{2}$, Chloë Roustan $^{2}$, Stephen R. Martin ${ }^{1}$, Peter B. Rosenthal ${ }^{3}$, John J. Skehel ${ }^{1}$, and Steven J. Gamblin*1

${ }^{1}$ Strutural Biology of Disease Processes Laboratory; ${ }^{2}$ Structural Biology Science Technology Platform; ${ }^{3}$ Structural Biology of Cells and Viruses Laboratory, Francis Crick Institute, NW1 1AT London, United Kingdom. ${ }^{4}$ Precision Medicine Center, The Seventh Affiliated Hospital, Sun Yat-sen University, Shenzhen, 518107, Guangdong, China

\# equal contribution

* to whom correspondence should be addressed: antoni.wrobel@crick.ac.uk, donald.benton@,crick.ac.uk, steve.gamblin@crick.ac.uk

Coronaviruses of bats and pangolins are implicated in the origin and evolution of the pandemic SARS-CoV-2. We show that Spikes from Pangolin-CoVs, closely related to SARS-CoV-2, bind strongly to human and pangolin ACE2 receptors. We also report Cryo-EM structure of Pangolin-CoV S and show it adopts a fully-closed conformation and that, aside from the Receptor-Binding Domain, it resembles the spike of a bat coronavirus RaTG13 more than that of SARS-CoV-2.

Despite intensive research into the origins of the COVID-19 pandemic, the evolutionary history of its causative agent SARS-CoV-2 remains unclear ${ }^{1,2}$. SARSCoV-2 belongs to the subgenus of sarbecoviruses, for which horseshoe bats (Rhinolophus sp.) are the reservoir species ${ }^{1,3,4}$. However, others have suggested ${ }^{5}$, and we recently demonstrated ${ }^{6}$, that the bat coronavirus RaTG13, the closest known relative of SARS-CoV-2, is unlikely to be able to infect human cells because of the very low affinity of its Spike protein for the human receptor ACE2. For this reason, it has been speculated that SARS-CoV-2 could have reached the human population via an intermediate host ${ }^{5}$. A number of recent studies reported the existence of sarbecoviruses highly similar to SARS-CoV-2 in diseased Malayan pangolins (Manis javanica) and 
thus pangolins were proposed to have played a role in the emergence of the current pandemic $^{7-10}$.

\section{The affinity of Pangolin-CoV S proteins for ACE2 receptors}

To characterise the pangolin virus Spike and compare it with that of SARS-CoV2, we expressed and purified two different Pangolin-CoV Spike ectodomains. These are based on the sequences of viruses isolated from pangolins seized in China's Guangdong province in $2019^{8,9}$. We also produced recombinant ectodomains of ACE2 proteins from human, bat (Rhinolophus ferremequinum) and pangolin in order to perform comparative biolayer interferometry assays. Both pangolin proteins (referred to as Pangolin-CoV S and Pangolin-CoV S') showed strong ( $<100 \mathrm{nM})$ binding towards the human ACE2, approximately ten-fold weaker binding to pangolin ACE2, and very weak binding to bat ACE2 (Fig. 1A and Fig. S1). A similar pattern of binding was observed for SARS-CoV$2 \mathrm{~S}$ (Fig. 1B); preferred and strong binding to human ACE2, weaker binding to pangolin ACE2 and very weak binding to bat ACE2.

These binding data demonstrate functional similarity between the RBDs of the $\mathrm{S}$ proteins from Pangolin-CoV and SARS-CoV-2, that correlates with the high degree of sequence identity, and three-dimensional structure similarity, between them (Fig 1D). None of the three species of ACE2 were bound strongly by the bat virus RaTG13 S (data not shown). This observation correlates with the substantial sequence differences between the RBD of RaTG13 and the RBDs of the other two species (Fig. 1D).

\section{Cryo-EM structure of Pangolin-CoV S}

We have determined the structure of the Pangolin-CoV S protein at $2.9 \AA$ by Cryo-EM (Fig. 2 \& Fig. S2). The structure is of similar resolution to our recent structure of SARS-CoV-2 $\mathrm{S}^{6}$, enabling a detailed comparison between the two. Overall, the structure of the Pangolin-CoV S (Fig. 2A) is similar to the closed form of the SARSCoV-2 and the RaTG13 S; the most striking feature is that all of the resolvable particles on the grid are in the closed conformation (compared with $83 \%$ in the uncleaved SARSCoV-2 S sample and $34 \%$ in the furin-cleaved in our previous study ${ }^{6}$ ). Comparison of the structures of $\mathrm{S}$ of Pangolin-CoV and SARS-CoV-2 identifies two specific, major differences that likely account for this feature.

Firstly, an amino-acid substitution in the otherwise highly conserved sequences in the interface between RBD neighbours in the $\mathrm{S}$ trimer, likely contributes to a more 
stable packing arrangement that favours the closed conformation (Fig. 2B). In detail, there is a salt bridge between in the closed form of SARS-CoV-2 formed by Lys417 and Glu406 in the RBD. In the Pangolin-CoV, an arginine is substituted at position 417 and, while it also makes a salt bridge with Glu406, the unique side-chain properties of the arginine residue induce different conformers at Arg403 and Tyr505 that enable additional stacking interactions and the formation of a hydrogen bond to the mainchain of Tyr369 in the neighbouring RBD. These interactions would be expected to contribute additional stabilisation to the RBD/RBD packing, hence favouring the closed form. Furthermore, in Pangolin-CoV S there are also two additional glycans close to the RBD interface (Fig. S4).

Secondly, the presence of a leucine residue at position 50 in the NTD-associated intermediate subdomain of Pangolin-CoV, compared with a serine residue in SARS$\mathrm{CoV}-2$, promotes a conformational arrangement that is further indicative of the closed form of S. Occupancy of a bulky, hydrophobic leucine (instead of the smaller, polar Ser) leads the helix (residues 294 to 304) to shift $1.5 \AA$ (to the right as viewed in Fig. 2C) compared with SARS-CoV-2, stabilising the formation of a helix-turn-helix structure between the two intermediate domains, which is not present in the SARS-CoV-2 but is present in RaTG13 S (Fig. 2C and Fig S3A). Folding of this motif has the effect of shifting the neighbouring RBD-associated subdomain as a rigid-body (to the left as viewed in Fig. 2D). A similar arrangement, of the helix-turn-helix, and rigid-body position of the domain are seen in the closed conformation of RaTG13 S (Fig. S3B). Moreover, analysis of the open conformations of S found in SARS-CoV-2 S shows that the RBD-associated intermediate domain shifts in the opposite direction upon S opening (Fig. 2D).

Taken together, these observations suggest several sequence-based differences, compared with SARS-CoV-2, that likely account for the Pangolin-CoV spike adopting an all-closed conformation. In an earlier work, we described the closed conformation adopted by the bat CoV RaTG13 S protein ${ }^{6}$. In that case, chemical crosslinking was required to stabilise the protein for Cryo-EM analysis, and so the possibility existed that the cross-linking had influenced its structure. The fact that the current closed conformation of pangolin-CoV S is remarkably similar, outside of the RBD, to the RaTG13 S suggests that the structure of the latter was probably not materially affected by the crosslinking. 
The likely role of the closed conformation for shielding the fusiogenic apparatus of S2 has been detailed in several places ${ }^{11-13}$, as with the need for the open conformation to facilitate receptor binding ${ }^{14,15}$. The similarity in affinity of intact SARS-CoV and SARS-CoV-2 Spike trimers and their isolated RBDs for ACE2 receptor ${ }^{16,17}$ implies that there is not a large energetic cost to opening of the S1 structure. Thus, the shielding effect of the closed conformation contributes to the stability of the trimer. When the virus encounters an ACE2-receptor-decorated cell surface, then the binding of ACE2 stabilises the open form of the Spike by inhibiting its re-closure. Thus, the presence of ACE2 receptors likely stimulates the opening of $S$ and its priming for subsequent membrane fusion.

\section{Conclusion}

While the non-RBD component of the S protein is very similar between SARSCoV-2 and the bat virus RaTG13 (96\% identity within S1), the sequence (97\% identity) and structure (RMSD $0.35 \AA$, Fig. 1D) of the RBD of SARS-CoV-2, in contrast, is remarkably similar to that of Pangolin-CoV, particularly at the ACE2 binding site (sequence identity between SARS-CoV-2 and RaTG13 is just $76 \%$ in this region). This close similarity of RBDs between Pangolin-CoV and SARS-CoV-2 correlates with the near identical binding properties of their two S proteins (Fig. 1). This suggests that, even though Pangolin-CoV and SARS-CoV-2 have significant sequence differences beyond their RBDs, especially in the NTD which for the Pangolin-CoV Spikes resembles more that of bat viruses ZXC21 and ZC45 than RaTG13, pangolin viruses might well be capable of infecting humans. In contrast, given the immeasurably low affinity of bat RaTG13 S for human ACE2, it seems unlikely that at least this class of presumed precursor bat viruses would infect humans.

There are conflicting reports on whether the RBD of Pangolin-CoV S, while very similar in sequence to the RBD of the current pandemic virus, is the immediate precursor to the SARS-CoV-2 RBD ${ }^{18,19}$. Our results suggest that the effective zoonotic range for this class of coronaviruses, beyond bats, may include species that, like pangolins, have ACE2 receptors similar to the human ACE2. Consequently, there are likely to be other, as yet unidentified, viruses that harbour RBDs of similar sequence and binding properties to SARS-CoV-2 and Pangolin-CoV. The existence of such RBDs in the relevant zoonotic background might account for the emergence of SARS-CoV-2 possibly via a recombination of bat viruses similar to RaTG13 with viruses perhaps not 
134 dissimilar to Pangolin-CoV. It is also important to note that various species of bat, even 135 within Rhinolophus genus, show considerable differences in their ACE2 sequences and 136 that it has not been possible to demonstrate direct binding of spike proteins from the 137 viruses most closely related to SARS-CoV-2 to bat ACE2. Thus, perhaps S of bat viruses 138 bind a different, as yet unidentified, cellular receptor(s). 

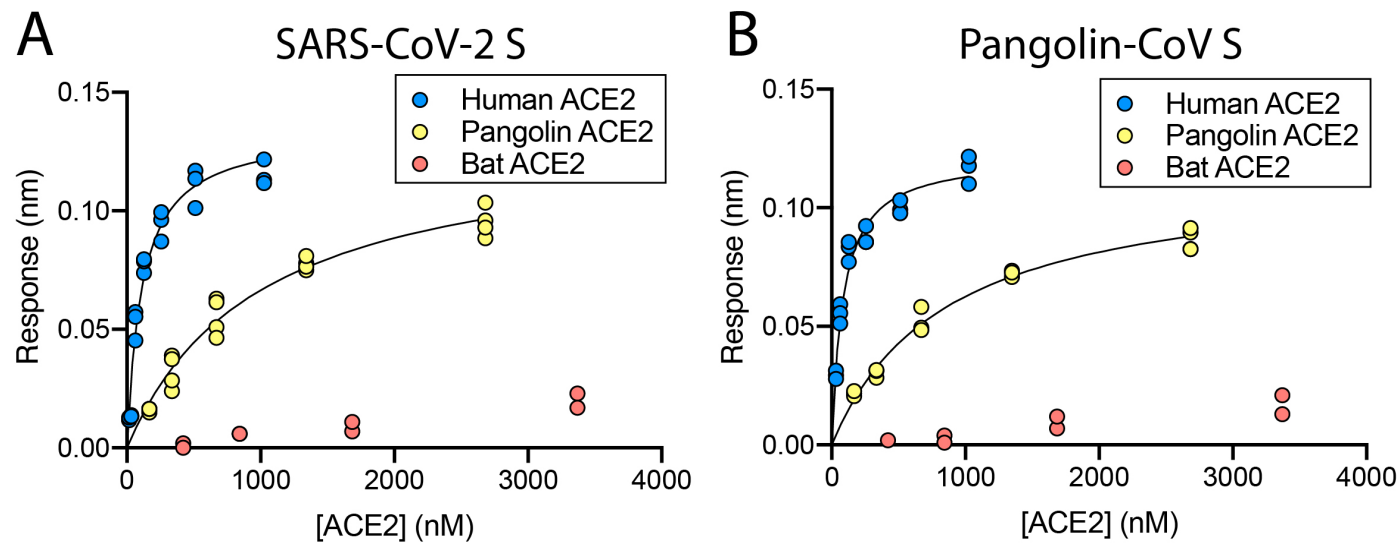

$\mathrm{D}$

\begin{tabular}{cccc} 
Spike & ACE2 & $\boldsymbol{K}_{\mathrm{d}}$ Amplitude $(\mathrm{nM})$ & $\boldsymbol{K}_{\mathrm{d}}$ Kinetics $(\mathrm{nM})$ \\
\hline \multirow{2}{*}{ SARS-CoV-2 } & Human & $110 \pm 14.7$ & $75.5 \pm 12.9$ \\
& Pangolin & $987 \pm 112$ & $896 \pm 225$ \\
\hline \multirow{2}{*}{ Pangolin-CoV } & Human & $74.0 \pm 13.0$ & $42.1 \pm 10.0$ \\
& Pangolin & $850 \pm 169$ & $663 \pm 139$
\end{tabular}

\begin{tabular}{|c|c|c|c|}
\hline 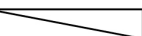 & SARS-CoV-2 & Pangolin-CoV & RaTG13 \\
\hline SARS-CoV-2 & & 0.35 & 0.87 \\
\hline Pangolin-CoV & 96.5 & & 0.62 \\
\hline RaTG13 & 89.5 & 89.0 & \\
\hline
\end{tabular}
species. (A,B) Plots of biolayer interferometry amplitudes for human (blue), pangolin (yellow) and bat (red) ACE2s binding to SARS-CoV-2 S (A) and pangolin-CoV S (B). (C) Equilibrium dissociation constants determined from the analysis of the data in $\mathbf{A}$ and $\mathbf{B}$ ( $\mathrm{K}_{\mathrm{d}}$ Amplitude) compared with values determined from analysis of the corresponding kinetic data ( $\mathrm{K}_{\mathrm{d}}$ Kinetics) (see Fig. S1). (D) Right hand side: RMSD of atom positions in the structures of RaTG13 S (6ZGF) ${ }^{6}$, closed conformation of SARSCoV-2 S (6ZGE) ${ }^{6}$, and Pangolin-CoV S RBD determined in this study. Left hand-side: sequence identity of the RBDs from the same viruses. 
A
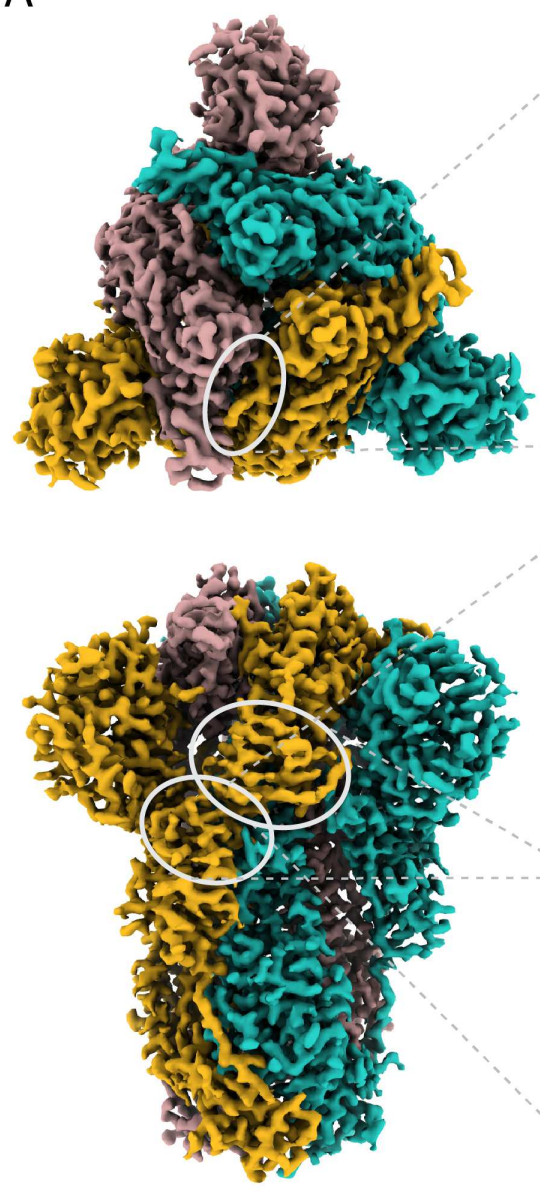

B

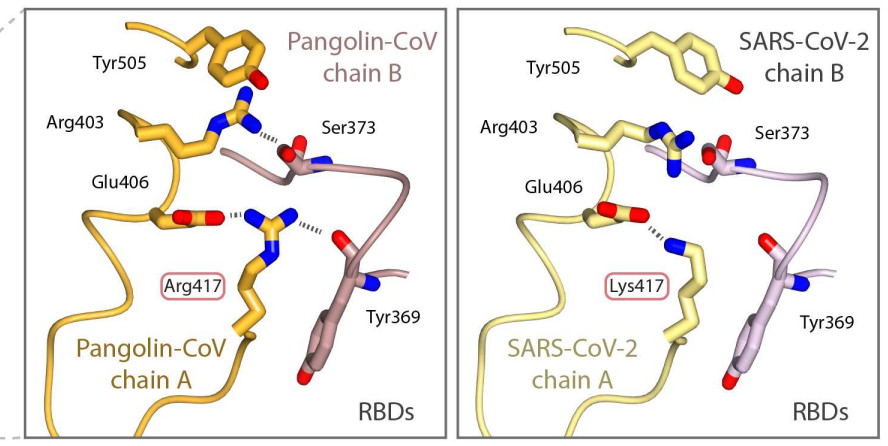

C
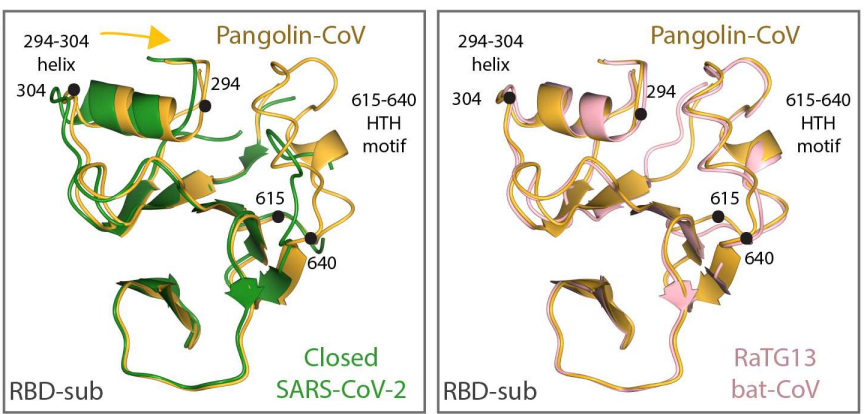

D

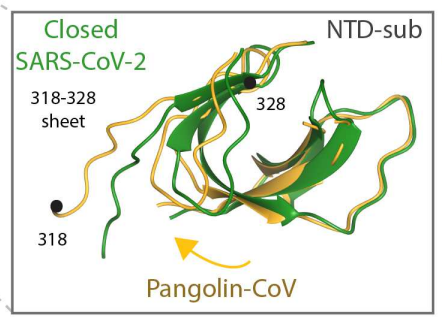

Figure 2: Structure of Pangolin-CoV Spike protein. (A) EM density representation from the $2.9 \AA$ map of Pangolin-CoV S viewed from down the three-fold axis (top panel) and in the orthogonal view (lower panel). The subunits are coloured in blue, golden and brown. The white ovals identify the areas shown in molecular representation on the right. (B) Comparison of the RBD/RBD interface from the pangolin-CoV (left) and SARS-CoV-2 S highlighting the Arg417Lys substitution. (C) Comparison of the RBDassociated subdomains of the pangolin-CoV (golden) and closed form of SARS-CoV-2 (green) in the left hand panel, showing the different positioning of the 294-304 helix and the presence of the 615-640 helix-turn-helix in the pangolin structure and, in the right-hand panel, the overlap of the same Pangolin-CoV S structure (golden) with the corresponding region from the RaTG13 (pink). (D) Comparison of (left) the NTDassociated subdomain of Pangolin-CoV (golden) with that of the closed form of SARSCoV-2 (green) showing the different domain orientations between them; (right) the closed (green) and open (blue) conformations of the NTD-associated subdomain of SARS-CoV-2 showing that the shift in orientation of the NTD-associated subdomain on Spike opening is in the opposite direction to the shift seen between the Pangolin-CoV and closed SARS-CoV-2 conformations shown in the left panel. 

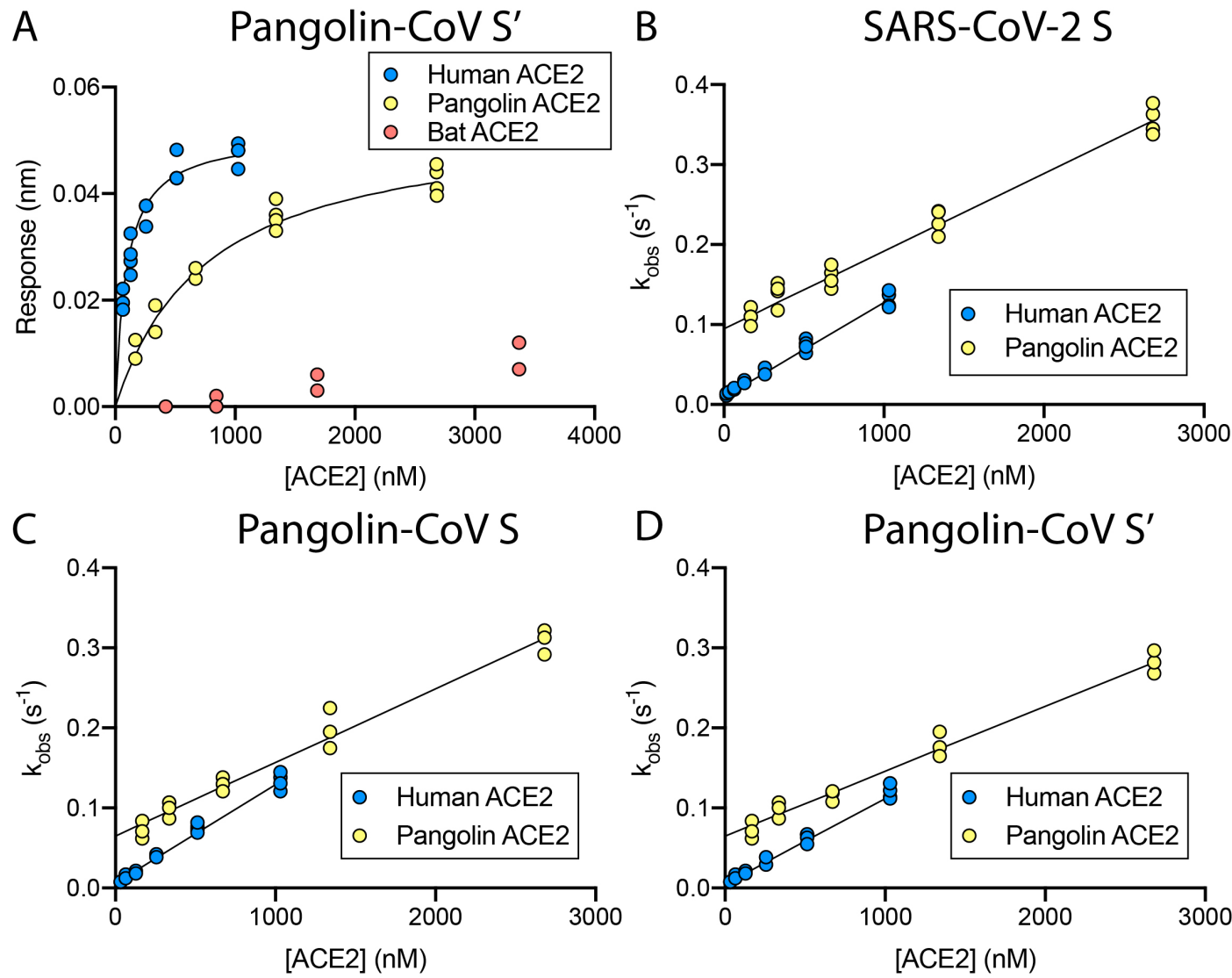

E

\begin{tabular}{clcccc} 
Spike & ACE2 & $\mathbf{K}_{\text {on }}\left(\mathrm{M}^{-1} \mathrm{~s}^{-1}\right)$ & $\mathbf{k}_{\text {off }}\left(\mathrm{s}^{-1}\right)$ & $\mathbf{K}_{\mathbf{d}}$ Kinetic $(\mathrm{nM})$ & $\mathbf{K}_{\mathrm{d}}$ Amplitude $(\mathrm{nM})$ \\
\hline \multirow{2}{*}{ SARS-CoV-2 S } & Human & $1.19 \times 10^{5} \pm 3.70 \times 10^{3}$ & $8.99 \times 10^{-3} \pm 1.49 \times 10^{-3}$ & $75.5 \pm 12.9$ & $110.0 \pm 14.7$ \\
& Pangolin & $9.70 \times 10^{4} \pm 6.80 \times 10^{3}$ & $8.70 \times 10^{-2} \pm 2.10 \times 10^{-2}$ & $896 \pm 225$ & $987 \pm 112$ \\
\hline \multirow{2}{*}{ Pangolin-CoV S } & Human & $1.24 \times 10^{5} \pm 7.00 \times 10^{3}$ & $5.22 \times 10^{-3} \pm 1.20 \times 10^{-3}$ & $42.1 \pm 10.0$ & $74.0 \pm 13.0$ \\
& Pangolin & $9.20 \times 10^{4} \pm 1.00 \times 10^{4}$ & $6.10 \times 10^{-2} \pm 1.10 \times 10^{-2}$ & $663.0 \pm 140$ & $850 \pm 169$ \\
\hline \multirow{2}{*}{ Pangolin-CoV S' } & Human & $1.08 \times 10^{5} \pm 1.50 \times 10^{4}$ & $5.90 \times 10^{-3} \pm 1.50 \times 10^{-3}$ & $54.6 \pm 15.8$ & $82.0 \pm 18.0$ \\
& Pangolin & $8.10 \times 10^{4} \pm 1.10 \times 10^{4}$ & $5.20 \times 10^{-2} \pm 1.70 \times 10^{-2}$ & $642.0 \pm 227$ & $970.0 \pm 160$
\end{tabular}

Figure S1: Biolayer interferometry on Spikes binding to ACE2s from different species. (A) Plots of biolayer interferometry amplitudes for human (blue), pangolin (yellow) and bat (red) ACE2s binding to pangolin-CoV S'. (B, C, D) Plots of observed rate constants for human (blue) and pangolin (yellow) ACE2s binding to SARS-CoV-2 S (B), Pangolin-CoV S (C) and Pangolin-CoV S' (D). (E) Association rate constants, dissociation rate constants and equilibrium dissociation constants. 


\section{A}
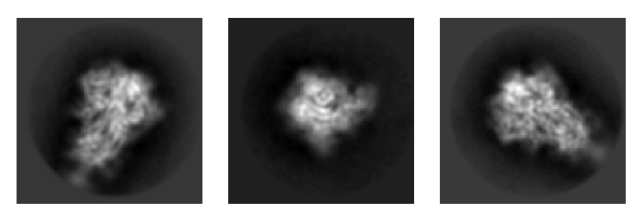

RELION
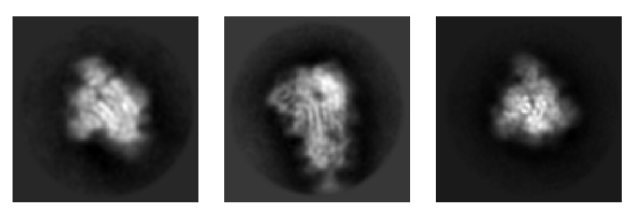

2D Classification $\times 2$
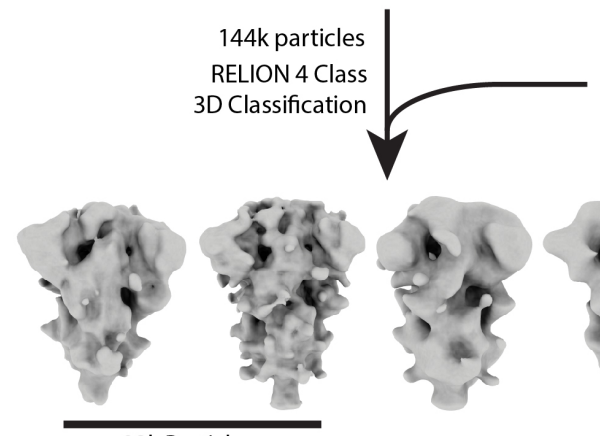

Low-pass filtered

Inital Model

from cryoSPARC

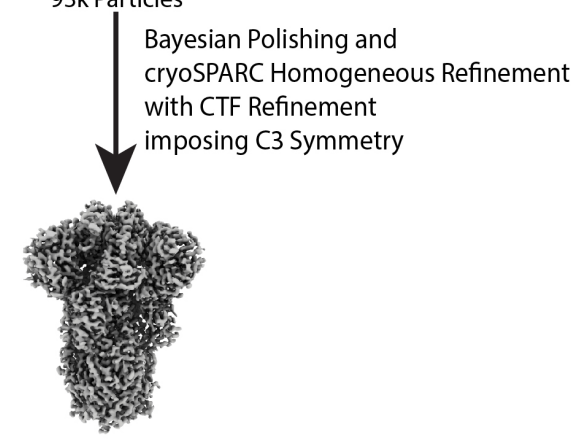

2.9 Å Resolution

B

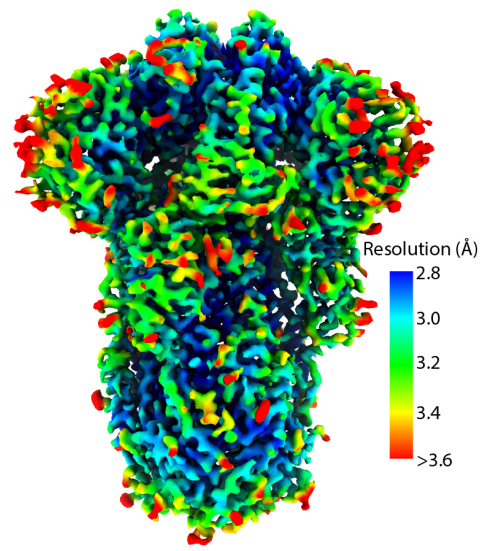

C

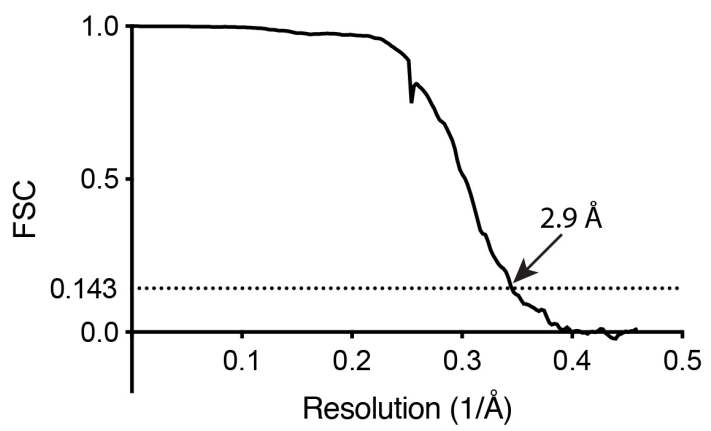

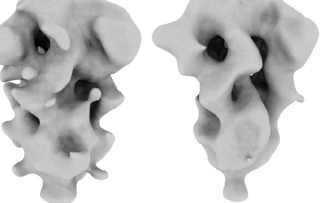

$$
\text { (1) }
$$




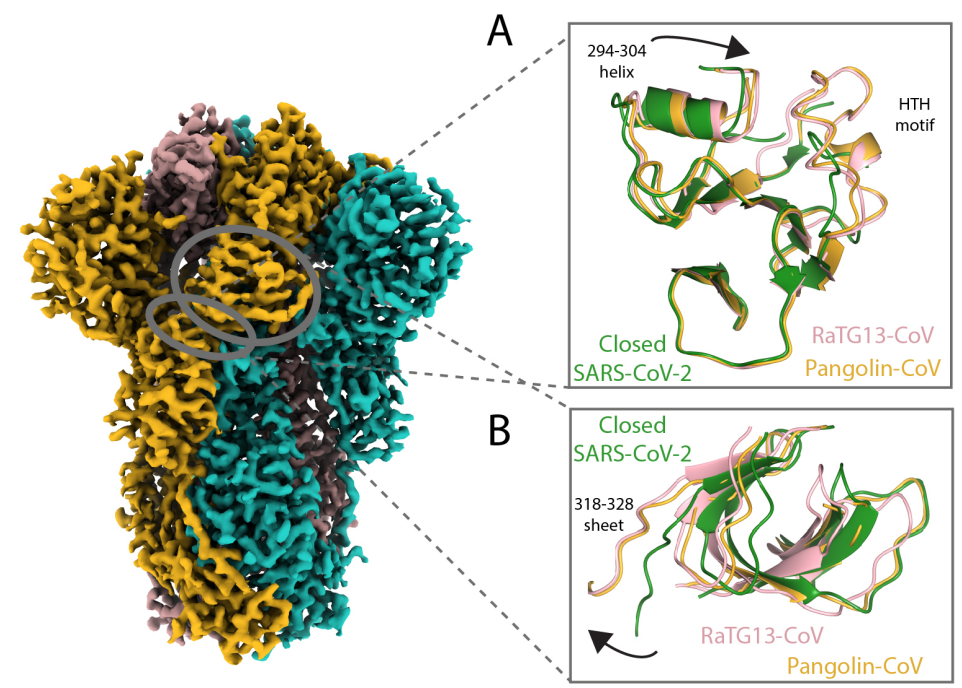

C

Figure S3: Comparison of Pangolin-CoV S with those from RaTG13 and SARSCoV-2. (A, B) Panels corresponding to Fig. 2A, 2C (A) and 2D (B). (C) Molecular model of the Pangolin-CoV S protein coloured with monomers in blue, golden, and brown in ribbon representation and substitutions between the Pangolin-CoV $\mathrm{S}$ and SARS-CoV-2 S shown as spheres in: green (for NTD), light plum (for NTD- and RBDassociated subdomains), red (for RBDs), and navy (S2). 


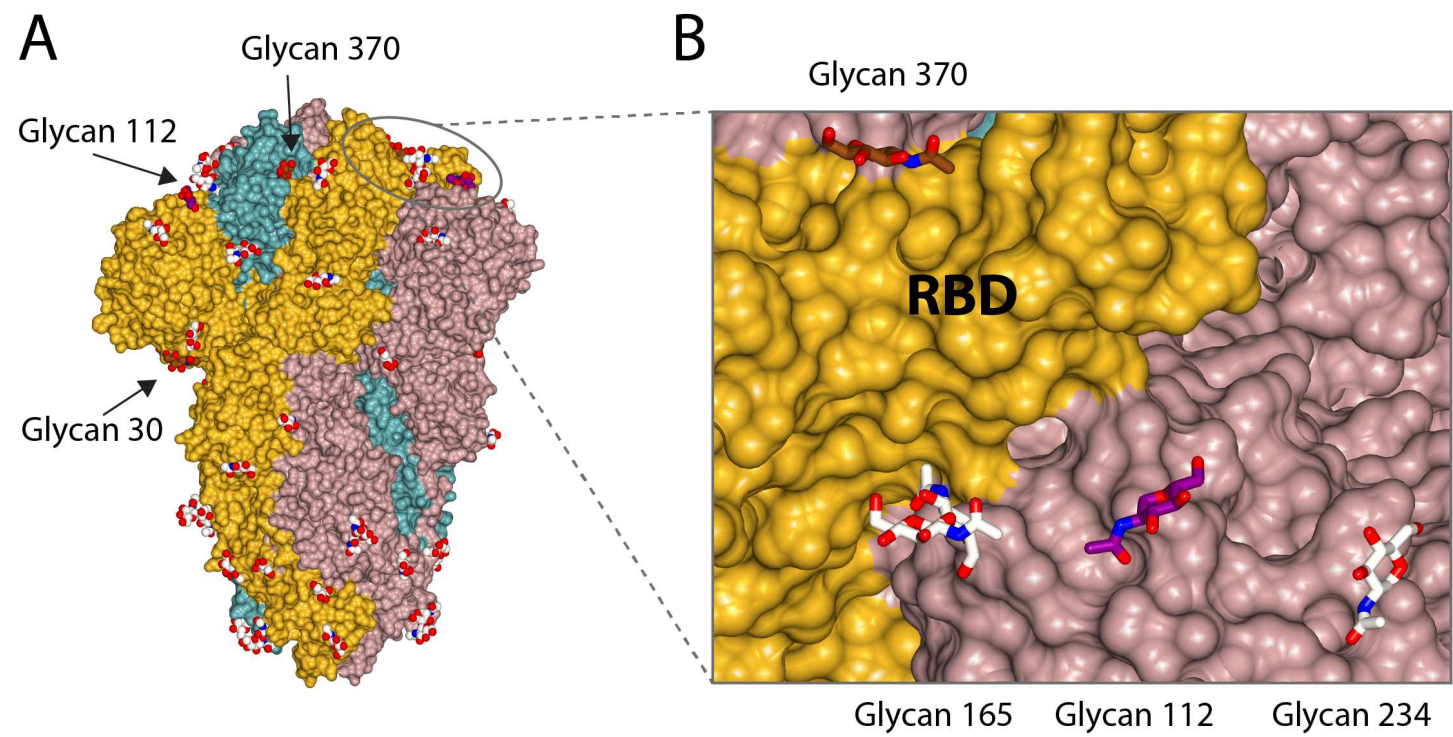

Figure S4: N-linked glycosylation of Pangolin-CoV S protein. (A) All glycans present in the Cryo-EM structure of the Pangolin-CoV S shown in balls representation, with those present in RaTG13 S and Pangolin-CoV S, but not SARS-CoV-2, shown in brown and that on Asn112, present uniquely in Pangolin-CoV, in purple. (B) Glycans on residues 165 and 234 suggested to be important for either inhibiting or enhancing the RBD erection ${ }^{20}$ shown in pale cream together with the two other glycans 370 and 112 , not present in SARS-CoV-2 spike, in proximity of the RBD. 
A.G.W, D.J.B, P.X, A.B., C.R, S.R.M performed research, collected and analysed data; A.G.W, D.J.B, P.B.R, J.J.S, S.J.G conceived and designed research and wrote the paper.

\section{Conflict Statement}

We have no conflicts of interest to declare.

211

\section{Data Availability}

The map and model have been deposited in the Electron Microscopy Data Bank, http://www.ebi.ac.uk/pdbe/emdb/ (Accession Nos. XXX,XXX,...). The model has been deposited in the Protein Data Bank, https://www.ebi.ac.uk/pdbe/ (PDB ID codes XXX, $\mathrm{XXX}, . .$.$) . [Accession numbers will be available before publication].$

\section{Acknowledgements}

We would like to acknowledge Andrea Nans of the Structural Biology Science Technology Platform for assistance with data collection, Phil Walker and Andrew Purkiss of the Structural Biology Science Technology Platform and the Scientific Computing Science Technology Platform for computational support. We thank Lesley Calder, Peter Cherepanov, George Kassiotis and Svend Kjaer for discussions. This work was funded by the Francis Crick Institute which receives its core funding from Cancer Research UK (FC001078 and FC001143), the UK Medical Research Council (FC001078 and FC001143), and the Wellcome Trust (FC001078 and FC001143). P.X. is also supported by the 100 Top Talents Program of Sun Yat-sen University, the Sanming Project of Medicine in Shenzhen (SZSM201911003) and the Shenzhen Science and Technology Innovation Committee (Grant No. JCYJ20190809151611269). 


\section{References}

1. Zhou, P. et al. A pneumonia outbreak associated with a new coronavirus of probable bat origin. Nature 579, 270-273 (2020).

2. Wu, F. et al. A new coronavirus associated with human respiratory disease in China. Nature 579, 265-269 (2020).

3. Li, W. et al. Bats are natural reservoirs of SARS-like coronaviruses. Science (80-. ). 310, 676-679 (2005).

4. Yang, L. et al. Novel SARS-like betacoronaviruses in bats, China, 2011. Emerg. Infect. Dis. 19, 989-991 (2013).

5. Andersen, K. G., Rambaut, A., Lipkin, W. I., Holmes, E. C. \& Garry, R. F. The proximal origin of SARS-CoV-2. Nature Medicine 26, 450-452 (2020).

6. Wrobel, A. G. et al. SARS-CoV-2 and bat RaTG13 spike glycoprotein structures inform on virus evolution and furin-cleavage effects. Nat. Struct. Mol. Biol. 1-5 (2020). doi:10.1038/s41594-020-0468-7

7. Lam, T. T. Y. et al. Identifying SARS-CoV-2 related coronaviruses in Malayan pangolins. Nature 1-6 (2020). doi:10.1038/s41586-020-2169-0

8. Xiao, K. et al. Isolation of SARS-CoV-2-related coronavirus from Malayan pangolins. Nature 583, 286-289 (2020).

9. Liu, P. et al. Are pangolins the intermediate host of the 2019 novel coronavirus (SARS-CoV-2)? PLOS Pathog. 16, e1008421 (2020).

10. Zhang, T., Wu, Q. \& Zhang Correspondence, Z. Probable Pangolin Origin of SARS-CoV-2 Associated with the COVID-19 Outbreak In Brief. Curr. Biol. 30, 1346-1351.e2 (2020).

11. Cai, Y. et al. Distinct conformational states of SARS-CoV-2 spike protein. Science (80-. ). eabd4251 (2020). doi:10.1126/science.abd4251

12. Li, F. Structure, Function, and Evolution of Coronavirus Spike Proteins. Annu. Rev. Virol. (2016). doi:10.1146/annurev-virology-110615-042301

13. Walls, A. C. et al. Tectonic conformational changes of a coronavirus spike glycoprotein promote membrane fusion. Proc. Natl. Acad. Sci. U. S. A. (2017). doi:10.1073/pnas.1708727114

14. Wrapp, D. et al. Cryo-EM structure of the 2019-nCoV spike in the prefusion conformation. Science (80-. ). (2020). doi:10.1126/science.aax0902

15. Walls, A. C. et al. Structure, Function, and Antigenicity of the SARS-CoV-2 Spike Glycoprotein. (2020). doi:10.1016/j.cell.2020.02.058

16. Shang, J. et al. Structural basis of receptor recognition by SARS-CoV-2. Nature 1-4 (2020). doi:10.1038/s41586-020-2179-y

17. Lan, J. et al. Structure of the SARS-CoV-2 spike receptor-binding domain bound to the ACE2 receptor. Nature (2020). doi:10.1038/s41586-020-2180-5

18. Boni, M. F. et al. Evolutionary origins of the SARS-CoV-2 sarbecovirus lineage responsible for the COVID-19 pandemic. Nat. Microbiol. 1-10 (2020). doi:10.1038/s41564-020-0771-4

19. Li, X. et al. Emergence of SARS-CoV-2 through recombination and strong purifying selection. Sci. $A d v$. eabb9153 (2020). doi:10.1126/sciadv.abb9153

20. Henderson, R. et al. Glycans on the SARS-CoV-2 Spike Control the Receptor Binding Domain Conformation. bioRxiv Prepr. Serv. Biol. 2020.06.26.173765 (2020). doi:10.1101/2020.06.26.173765 


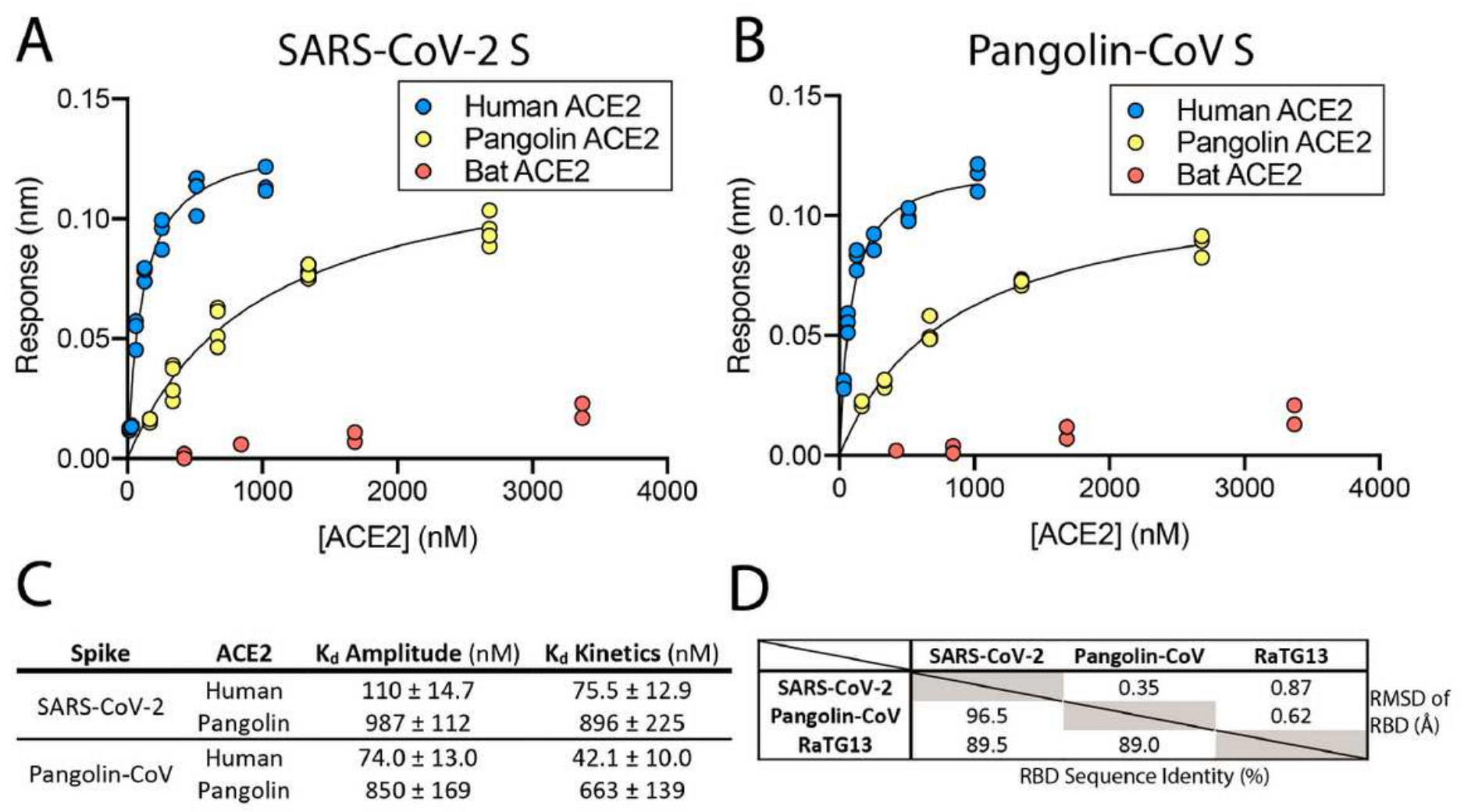

\section{Figure 1}

Binding of Pangolin-CoV and SARS-CoV-2 S to ACE2s from different species. (A,B) Plots of biolayer interferometry amplitudes for human (blue), pangolin (yellow) and bat (red) ACE2s binding to SARS-CoV$2 S$ (A) and pangolin-CoV S (B). (C) Equilibrium dissociation constants determined from the analysis of the data in $A$ and $B$ ( $K d$ Amplitude) compared with values determined from analysis of the corresponding kinetic data (Kd Kinetics) (see Fig. S1). (D) Right hand side: RMSD of atom positions in the structures of RaTG13 S (6ZGF)6, closed conformation of SARS CoV-2 S (6ZGE)6, and Pangolin-CoV S RBD determined in this study. Left hand-side: sequence identity of the RBDs from the same viruses. 


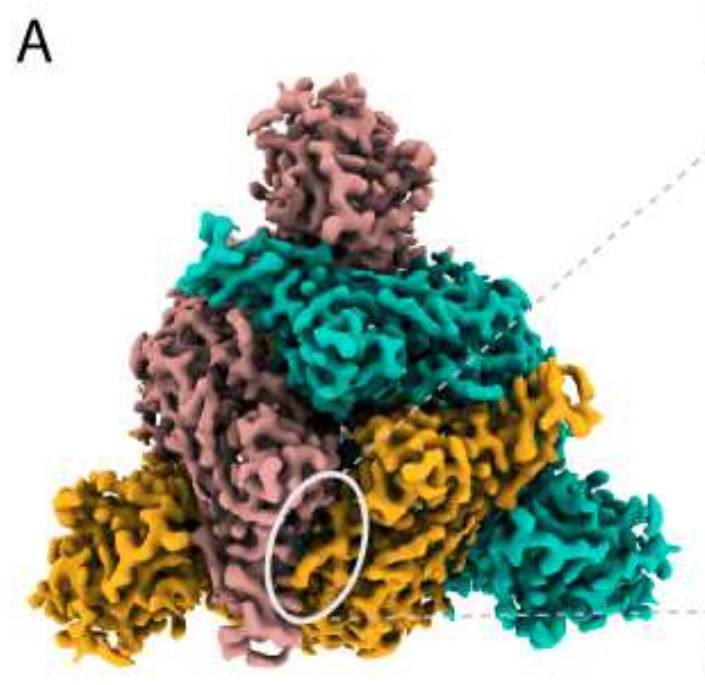

B

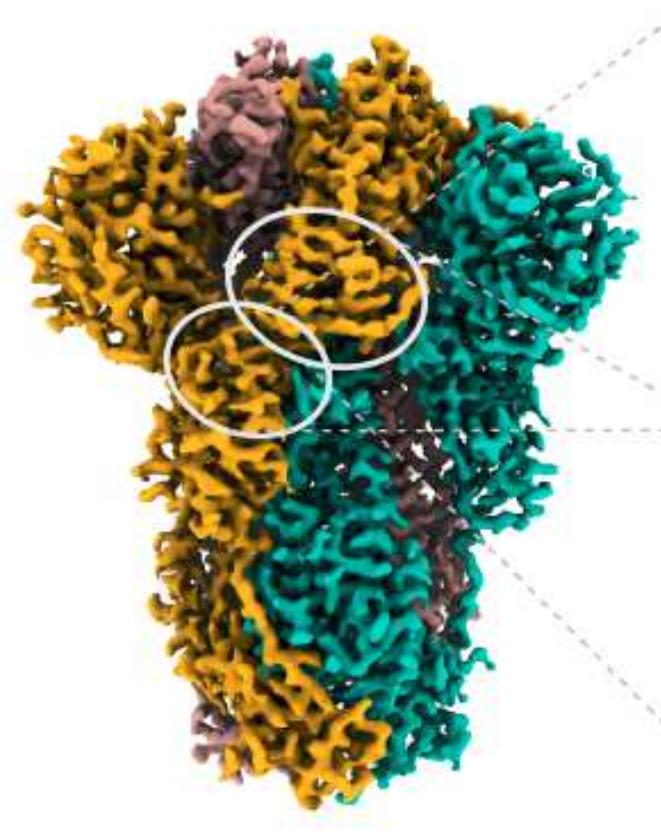

\section{C}
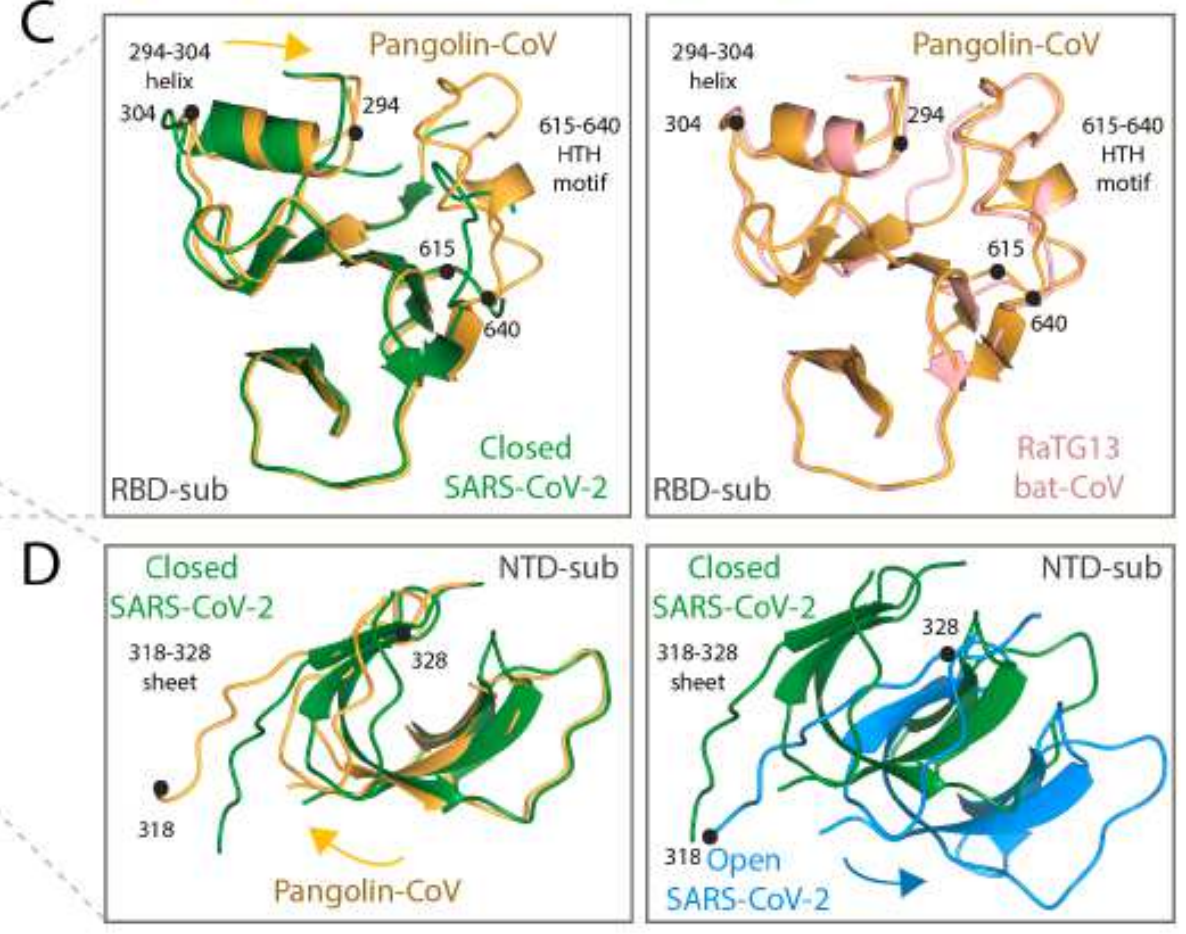

Figure 2

Structure of Pangolin-CoV Spike protein. (A) EM density representation from the $2.9 \AA$ map of PangolinCoV S viewed from down the three-fold axis (top panel) and in the orthogonal view (lower panel). The subunits are coloured in blue, golden and brown. The white ovals identify the areas shown in molecular representation on the right. (B) Comparison of the RBD/RBD interface from the pangolin-CoV (left) and SARS-CoV-2 S highlighting the Arg417Lys substitution. (C) Comparison of the RBD associated subdomains of the pangolin-CoV (golden) and closed form of SARS-CoV-2 (green) in the left hand panel, showing the different positioning of the 294-304 helix and the presence of the 615-640 helix-turn-helix in the pangolin structure and, in the right-hand panel, the overlap of the same Pangolin-CoV S structure (golden) with the corresponding region from the RaTG13 (pink). (D) Comparison of (left) the NTD associated subdomain of Pangolin-CoV (golden) with that of the closed form of SARS CoV-2 (green) showing the different domain orientations between them; (right) the closed (green) and open (blue) 
conformations of the NTD-associated subdomain of SARS-CoV-2 showing that the shift in orientation of the NTD-associated subdomain on Spike opening is in the opposite direction to the shift seen between the Pangolin-CoV and closed SARS-CoV-2 conformations shown in the left panel. 